Nano LIFE

Vol. 4, No. 4 (2014) 1441014 (13 pages)

(C) World Scientific Publishing Company

DOI: $10.1142 /$ S1793984414410141

\title{
Effects of Size and Dispersity of Microcrystalline Celluloses on Size, Structure and Stability of Nanocrystalline Celluloses Extracted by Acid Hydrolysis
}

\author{
Qi Liu \\ National Engineering Laboratory for Crop Efficient Water Use \\ and Disaster Mitigation and Key Laboratory of Dryland Agriculture \\ Ministry of Agriculture, Institute of Environment \\ and Sustainable Development in Agriculture \\ Chinese Academy of Agricultural Sciences, Beijing 100081, P. R. China \\ Department of Agriculture, Bio-engineering and Chemistry \\ University of Liege-Gembloux Agro-Bio Tech, 5030 Belgium \\ liuqi@caas.cn \\ Weiping Hao \\ National Engineering Laboratory for Crop Efficient Water Use \\ and Disaster Mitigation and Key Laboratory of Dryland Agriculture \\ Ministry of Agriculture, Institute of Environment \\ and Sustainable Development in Agriculture \\ Chinese Academy of Agricultural Sciences, Beijing 100081, P. R. China \\ haoweiping@caas.cn \\ Yongguang Yang \\ Department of Cancer and Cell Biology \\ University of Cincinnati College of Medicine \\ Cincinnati, Ohio 45267, USA \\ yangy9@ucmail.uc.edu \\ Aurore Richel \\ Unit of Biological and Industrial Chemistry \\ University of Liege-Gembloux Agro-Bio Tech, 5030 Belgium \\ a.richel@ulg.ac.be \\ Canbin Ouyang \\ Department of Pesticide, Key Laboratory of Pesticide Chemistry \\ and Application, Institute of Plant Protection \\ Chinese Academy of Agricultural Sciences \\ Beijing 100193, P. R. China \\ oycb@iccas.ac.cn
}


Q. Liu et al.

\author{
Huan Liu \\ School of Music and Recording Arts \\ Communication University of China \\ Beijing 100024, P. R. China \\ Guor219@163.com \\ Rui Guo ${ }^{\star}$ and $\mathrm{Xu} \mathrm{Xia}{ }^{\S}$ \\ National Engineering Laboratory for Crop Efficient Water Use \\ and Disaster Mitigation, and Key Laboratory of Dryland Agriculture \\ Ministry of Agriculture \\ Institute of Environment and Sustainable Development in Agriculture \\ Chinese Academy of Agricultural Sciences, Beijing 100081, P. R. China \\ tguorui@caas.cn \\ §xiaxu@caas.cn \\ Jing Yang \\ Construction Engineering Test Center \\ Central Research Institute of Building and Construction CO., Ltd. \\ MCC Group, Beijing 100088, P. R. China \\ yj001010@126.com \\ Jiqing Song \\ National Engineering Laboratory for Crop Efficient Water Use \\ and Disaster Mitigation, and Key Laboratory of Dryland Agriculture \\ Ministry of Agriculture \\ Institute of Environment and Sustainable Development in Agriculture \\ Chinese Academy of Agricultural Sciences, Beijing 100081, P. R. China \\ songjiqing@caas.cn \\ Dorothée Goffin \\ Department of Agriculture, Bio-engineering and Chemistry \\ University of Liege-Gembloux Agro-Bio Tech, 5030 Belgium \\ dorothee.goffin@ulg.ac.be
}

Received 13 August 2014

Accepted 2 September 2014

Published 16 October 2014

\begin{abstract}
Nanocrystalline celluloses (NCCs) were separated from four commercial microcrystalline celluloses (MCCs) by an acid hydrolysis-sonication treatment. Transmission electron microscopy (TEM), atomic force microscopy (AFM), Fourier transform infrared (FTIR) spectrum, X-ray diffraction (XRD) and thermogravimetric analysis (TGA) were conducted to investigate the NCCs. MCCs with different morphologies and particle sizes showed different aggregation degrees. The aggregation of MCCs followed the order MCC1 > MCC3 > MCC2 > MCC4, which is the same order of the heights of the resulting NCCs. The best uniformity and thermal stability were characterized for NCC3, which was produced by MCC3 with smallest original particle size and good dispersity among the four MCCs. This result suggests that both the original particle size and dispersity of MCCs had significant effects on separated NCCs.
\end{abstract}

Keywords: Microcrystalline cellulose; acid hydrolysis; nanocrystalline celluloses.

TCorresponding authors. 


\section{Introduction}

Cellulose is an important biopolymer and the most ubiquitous and abundant natural biomacromolecule produced by plants and microorganisms worldwide. ${ }^{1}$ Cellulose fibers possess highly advantageous properties, such as low cost, biodegradability, renewability, high specific strength and modulus, low density, easy processability and relatively reactive surface for grafting specific groups. ${ }^{2}$ This material is widely used because of its biocompatibility, biological degradability, and sustainability. Microcrystalline celluloses (MCCs) represent novel commercial cellulose and exist as a fine, white, odorless, crystalline powder, which has been used for years in different industries, including cosmetics, food, pharmaceuticals and plastics. ${ }^{3}$ Numerous researchers have reported on the preparation and characterization of MCC particles isolated from various cellulosic sources. ${ }^{4-6}$

Producing composite materials with nanocrystalline celluloses (NCCs) reinforcement has been recently attracting increasing attention because of the extraordinary properties of the nanometric size effect for reinforcement. Stable NCC suspensions have been known for more than half a century to be prepared by submitting native cellulose to a harsh sulfuric acid hydrolysis and often followed by ultrasound treatments. ${ }^{7}$ NCCs prepared from different types of raw cellulose materials are characterized by methods, such as transmission electron microscopy (TEM), atomic force microscopy (AFM) and scanning electron microscopy (SEM). ${ }^{8,9}$ Particles are generally elongated and flat, a few hundreds of nanometers long, $10 \mathrm{~nm}$ to $20 \mathrm{~nm}$ wide, and a few nanometer thick. ${ }^{10,11}$ Several studies about preparation and characterization of NCCs from MCCs also exist. ${ }^{12,13}$ Compared with cellulose nanowhiskers extracted from industrial bioresidue, those isolated from MCCs by acid hydrolysis show higher surface charge and crystallinity, as well as lower aggregation and degradation temperature. ${ }^{12}$ The high crystallinity of these nanoscale celluloses makes these materials a suitable reinforcement for biocomposites. In addition, Bondeson et al. ${ }^{13}$ reported that obtaining cellulose nanocrystals/whiskers with a length between $200 \mathrm{~nm}$ and $400 \mathrm{~nm}$ and a width less than $10 \mathrm{~nm}$ is possible with a sulfuric acid concentration of $63.5 \%$ $(\mathrm{w} / \mathrm{w})$. The effects of hydrolysis conditions and surface charge density, ${ }^{14}$ ionic strength ${ }^{15}$ and $\mathrm{pH}^{16}$ on the phase separation behavior and structures of the products have been studied over the past two decades.

Although numerous studies about the effect of hydrolysis conditions and properties of the resulting NCCs have been conducted, comparative studies about acid hydrolysis of MCCs with different morphologies, particle sizes and dispersibilities are few. In this study, we submitted four commercial MCCs to sulfuric acid hydrolysis and examined their morphology and particle size. The shape and size distribution of the resulting NCCs were determined by TEM and AFM. Fourier transform infrared (FTIR) spectrum and X-ray diffraction (XRD) experiments were used to describe material structure and crystallinity. Thermogravimetric analysis (TGA) was conducted to determine thermal stability.

\section{Materials and Methods}

\subsection{Materials}

The four commercial MCCs used in this work were purchased from Ruibio-bio Company (Germany), Sigma-Aldrich Company (USA), Aladdin Industrial Corporation (China) and Xiya Regent Research Center (China). All other reagents and chemicals were of analytical grade. All experiments were performed with ultrapure water.

\subsection{Preparation of the NCCs}

MCCs were hydrolyzed according to the method described by Revol et al. ${ }^{17}$ All four samples were treated with $64 \%$ sulfuric acid at a temperature of $50^{\circ} \mathrm{C}$ in a Pro Thermo Shaker NTS-3100 (Rikakikai Co., Ltd, Tokyo, Japan) and a speed of $120 \mathrm{r} / \mathrm{min}$ for $40 \mathrm{~min}$. MCC content was $5 \mathrm{wt} . \%$. The bottles with suspension were placed in cold water to quench the reactions. The hydrolyzed samples were washed by centrifugation at $15000 \mathrm{rpm}$ and $8^{\circ} \mathrm{C}$ for $15 \mathrm{~min}$ with a Heraeus centrifuge (Heraeus Co., Ltd, Germany). Centrifugation was repeated five times before the suspension was dialyzed against ultrapure water for several days to neutrality. The molecular weight cut-off of the dialysis bag ranged from $8000 \mathrm{Da}$ to $14000 \mathrm{Da}$. The resulting suspension was then sonicated for 10 min with a JY99IIDN Ultrasonic Processor (Saifu Experimental Instrument Co., Ltd, Ningbo, China) at an output power of $1100 \mathrm{~W}$ to obtain the NCCs. The entire treatment process was performed in an ice bath. 
A portion of the each resulting suspension was then freeze-dried with an FD-1 freeze dryer at $-50^{\circ} \mathrm{C}$ (Boyikang Experimental Instrument Co., Ltd, Beijing, China) to obtain the NCC powder. The resulting products were then collected.

\subsection{Characterization}

\subsubsection{Morphology of MCCs}

The cellulose fibers were observed by SEM using a Hitachi S-4800 field emission scanning electron microscope (Hitachi High Technologies America, Inc., USA). The original MCC cellulose fibers were directly used. The samples were coated with gold by an ion sputter coater and were observed under SEM operating at $5 \mathrm{kV}$.

\subsubsection{Laser particle size analysis of MCCs}

The particle size distributions of all the MCC samples were analyzed using a Malvern Mastersizer 2000 laser-diffraction particle size analyzer (Malvern Instruments Ltd., UK) with Malvern Mastersizer 2000 software. The samples were first measured directly in the original state of aggregation to obtain the mean volume diameter of the aggregated particle size. The primary particle size of the MCCs was achieved by sonication for $10 \mathrm{~s}$. Aggregation degree was calculated from the ratio of the aggregated particle size to the primary particle size.

\subsubsection{Morphology and size of NCCs}

The morphology of the fibers was characterized with both TEM and AFM.

\subsubsection{TEM}

Drops of 0.001 wt.\% NCC suspensions were deposited on carbon-coated TEM grids. The samples were then negatively stained with $2 \%$ uranyl acetate prior to complete drying and observed under a JEM1200EX transmission electron microscope (JEOL Ltd., Tokyo, Japan) operating at $80 \mathrm{kV}$. Images were recorded.

\subsubsection{AFM}

To accurately characterize surface topography and microfibril structure, we obtained surface imaging and particle height distribution using a Mutimode 8 atomic force microscope (Bruker Daltonics Inc., USA). Drops of dilute cellulose NCC suspensions were deposited onto freshly cleaved mica and allowed to dry. AFM observations were carried out operating in nitrogen condition and using a ScanA syst mode. ScanA syst is based on the Bruker-exclusive Peak Force Tapping mode. Tapping mode is speculated to be more suitable than contact mode for imaging polymer surfaces because the AFM tip intermittently contacts the surface, leading to minimized destructive lateral forces that can produce surface artifacts. ${ }^{19}$ Therefore, all AFM images were recorded in tapping mode.

\subsubsection{FTIR spectroscopy}

FTIR spectroscopy was used to examine any change in the structure of the cellulose fibers after nanocrystallization treatment. A Thermo Scientific Nicolet iN10 (Thermo Electron Corp., USA) was employed to record the FTIR spectrum of each sample. The dried samples were ground and then blended with $\mathrm{KBr}$ before pressing the mixture into ultrathin pellets. Spectral outputs were recorded in the transmittance mode as a function of wave number.

\subsubsection{Powder XRD}

Crystallinities of the MCC and NCC fibers were examined using a Bruker D8 Focus (Bruker AXS Inc., Madison, WI, USA) equipped with a high power point focus $\mathrm{CuK} \alpha$ target, graphite monochromator for elimination of $\operatorname{Cuk} \beta$ lines, and a HiStar General Area Diffraction Detection System area detector for 2D images. High-resolution frames for accurate integration of the diffraction images were recorded. Scattered radiation was detected in the range of $2 \theta=10^{\circ}$ to $30^{\circ}$ at a scan rate of $4^{\circ} / \mathrm{min}$.

Crystallinity index (CI) was calculated from the heights of the 200 peak $\left(I_{200}, 2 \theta=22.6^{\circ}\right)$ and the intensity minimum is between the 200 and 110 peaks $\left(I_{\text {am }}, 2 \theta=18^{\circ}\right)$ with the Segal's method, ${ }^{20}$ as shown in Eq. (1). $I_{200}$ represents both crystalline and amorphous material, whereas $I_{\mathrm{am}}$ represents the amorphous material.

$$
\mathrm{CI}(\%)=\left(1-\frac{I_{\mathrm{am}}}{I_{200}}\right) \times 100
$$

\subsubsection{Thermal analysis}

TGA was performed to compare the degradation characteristics of MCCs with those of NCCs. The thermal stability of each sample was determined using a TGA/DSC 1 thermogravimetric analyzer 
(Mettler Toledo Corporation, Schwarzenbach, Switzerland) and analyzed by STAR software (Version 9.3). Runs were performed by weighing out the neat (bulk) surfactant ( $1 \mathrm{mg}$ to $4 \mathrm{mg}$ ) in a $70 \mu \mathrm{L}$ aluminum cup. The cup was crimped, and a hole was pierced automatically by the instrument just prior to the analysis. The analysis was performed with a constant flux of $40 \mathrm{~mL} / \mathrm{min}$ of nitrogen to ensure that the change in weight is due to thermal degradation. The temperature of the sample was ramped at a constant rate of $10^{\circ} \mathrm{C} / \mathrm{min}$, and weight loss or heat evolved was measured against increasing temperature.

\section{Results and Discussion}

\subsection{Morphology of MCCs}

The morphology of fiber or particle materials is usually observed by SEM. ${ }^{21-23}$ The MCC particles were investigated with SEM to determine their shape, size and surface morphology. Figure 1 is a comparison of the SEM images of each MCC prior to hydrolysis. The micrographs show the different shapes of the four MCCs. Figure 1(a) shows that MCC1 are ribbon-like in structure and are composed of long cellulose microfibrils. The microfibrils, which measured about $15 \mu \mathrm{m}$ to $25 \mu \mathrm{m}$ wide and $30 \mu \mathrm{m}$ to $200 \mu \mathrm{m}$ long, maintain the basic shape of cotton fiber. Figure 1(b) indicates that the particles of MCC2 are nonuniformly sized at about $10 \mu \mathrm{m}$ to
$100 \mu \mathrm{m}$. Figure 1(c) shows that MCC3 are more uniform and composed of smaller particles with diameters between $10 \mu \mathrm{m}$ and $50 \mu \mathrm{m}$. Figure $1(\mathrm{~d})$ indicates that the cellulose particles of MCC4 are larger than those of MCC3 and smaller than those of MCC2. The MCCs show diverse shape and size, but these micro-sized cellulose fibers were reported to be composed of strong hydrogen bonding nanofibers, ${ }^{24}$ which enable isolation of nanoscale cellulose fibers from the MCCs.

\subsection{Laser particle size analysis}

The size distribution of MCCs was investigated by comparing the mean volume diameter before and after sonication. The test results for each sample and the calculated aggregation degree are given in Table 1. Sonication of the original MCCs for $10 \mathrm{~s}$ resulted in a significant reduction in particle size distribution of the MCC sample, especially for MCC1. The different MCCs had different aggregation degrees, which followed the order MCC1 > MCC3 $>$ MCC2 > MCC4 as listed in Table 1. The strong aggregation showed by MCC1 may cause incomplete reaction during hydrolysis when preparing NCCs. However, MCC4 exhibited similar particle size before and after the sonication treatment. This result may be beneficial for sulfuric acid to access the cellulose molecule because MCCs were used to prepare NCCs without any treatment.
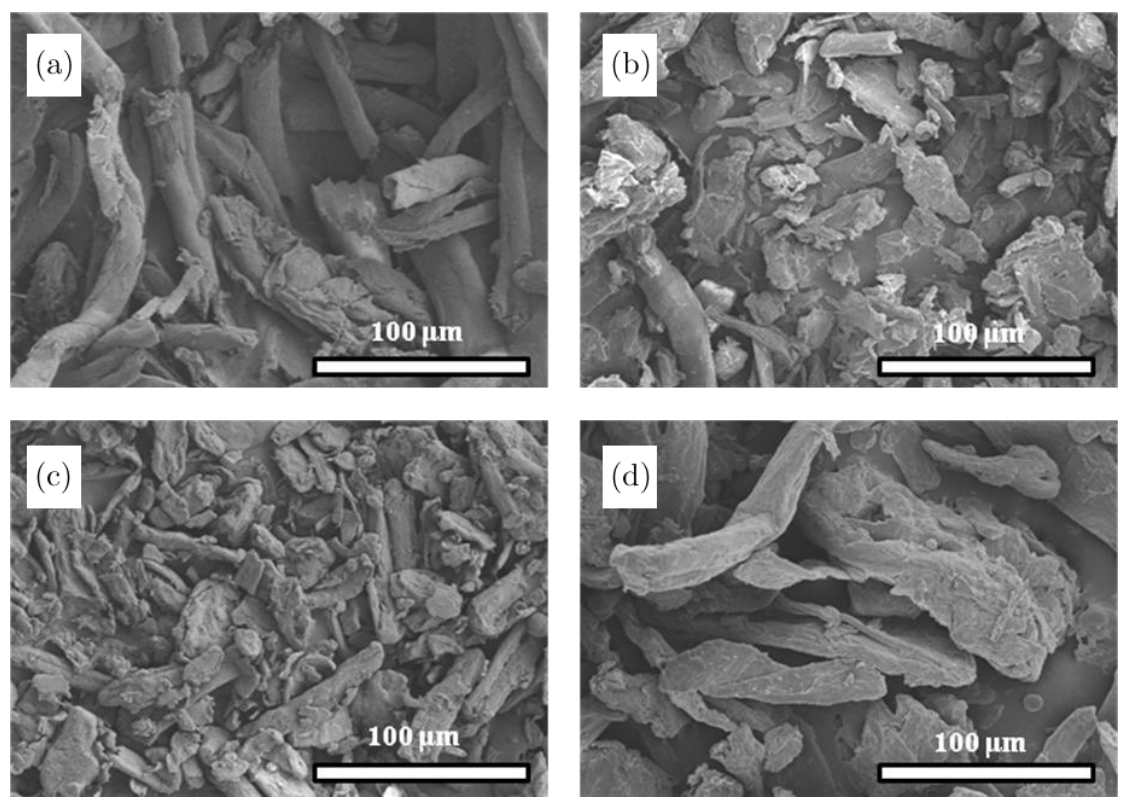

Fig. 1. SEM micrographs of the MCCs: (a) MCC1, (b) MCC2, (c) MCC3 and (d) MCC4. 
Table 1. Particle size and aggregation degree of MCCs.

\begin{tabular}{lccc}
\hline & \multicolumn{2}{c}{ Mean volume diameter $(\mu \mathrm{m})$} & \\
\cline { 2 - 3 } & $\begin{array}{c}\text { Before } \\
\text { sonication }\end{array}$ & $\begin{array}{c}\text { After } \\
\text { sonication }\end{array}$ & $\begin{array}{c}\text { Aggregation } \\
\text { degree }\end{array}$ \\
\hline MCC1 & $190.1 \pm 0.22$ & $40.0 \pm 0.14$ & 4.75 \\
MCC2 & $49.4 \pm 0.06$ & $33.5 \pm 0.67$ & 1.47 \\
MCC3 & $56.4 \pm 0.09$ & $29.9 \pm 0.06$ & 1.89 \\
MCC4 & $54.6 \pm 0.04$ & $50.2 \pm 0.12$ & 1.09 \\
\hline
\end{tabular}

Figure 2 presents the particle size distributions of the four MCCs. The size distribution of the samples after sonication showed that the mean volume diameters were $40.0 \mu \mathrm{m}, 33.5 \mu \mathrm{m}, 29.9 \mu \mathrm{m}$ and $50.2 \mu \mathrm{m}$, which is in accordance with the results shown in SEM micrographs. As shown in Figs. 2(b) and 2(c), MCC2 and MCC3 showed a main simple peak with a slight tail. However, the original particle distributions of MCC1 and MCC4 shown in Figs. 2(a) and $2(\mathrm{~d})$ present two or even more peaks. For MCC1, this result was mainly caused by the high aggregation degree of the original particles. MCC4 showed two main peaks at around $35 \mu \mathrm{m}$ and $100 \mu \mathrm{m}$, which may be due to the raw material species or the preparation technology.

\subsection{Morphology and size of NCCs}

\subsubsection{TEM}

Figure 3 shows TEM micrographs of all nanoparticles prepared by sulfuric acid hydrolysis of the four commercial MCCs. The nanoscale particles isolated from MCCs all present an elongated rod shape. The NCCs were approximately $100 \mathrm{~nm}$ to $250 \mathrm{~nm}$ long and $10 \mathrm{~nm}$ to $30 \mathrm{~nm}$ wide. NCC4 showed the thinnest width at around $10 \mathrm{~nm}$, whereas all the other three ranged from $20 \mathrm{~nm}$ to $25 \mathrm{~nm}$. An evident whisker fraction was observed in Fig. 3(b), which is possibly caused by localized damage created during the process of sonication treatment. ElazzouziHafraoui et al. ${ }^{18}$ also found this phenomenon in their study of acid hydrolysis of tunicin whiskers. Each image of the samples clearly revealed by negative staining that most particles were composed of several parallel elongated rods. Given the significant

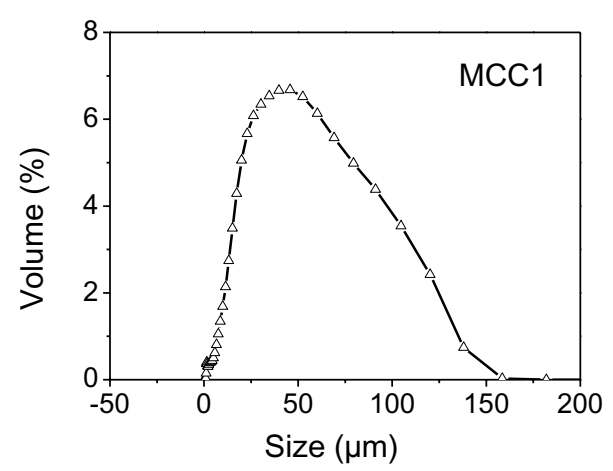

(a)

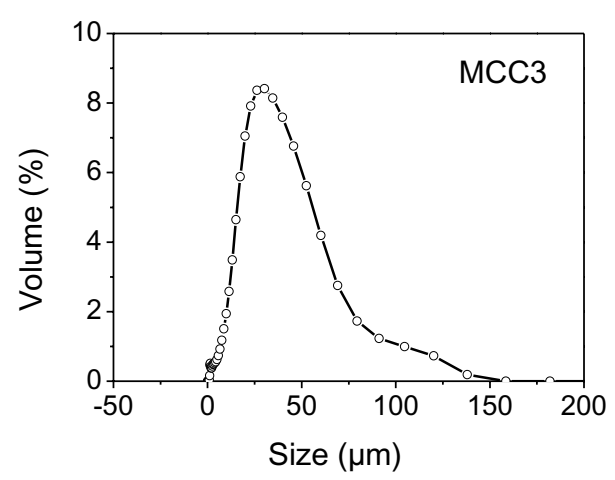

(c)

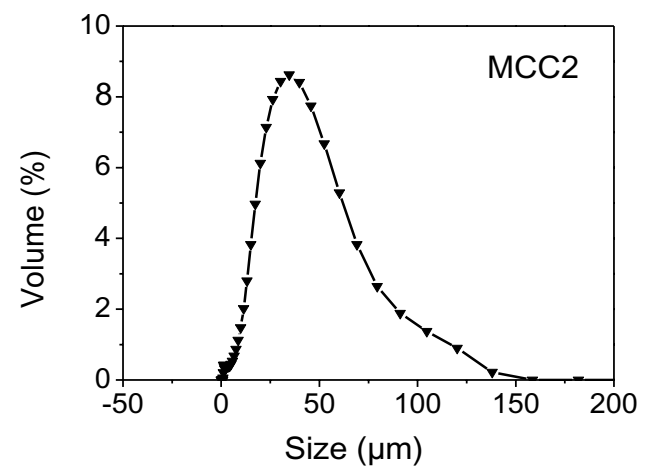

(b)

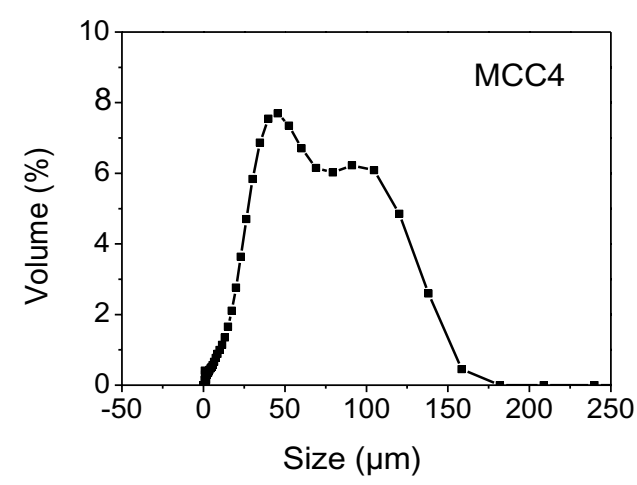

(d)

Fig. 2. Particle size distribution of the MCCs: (a) MCC1, (b) MCC2, (c) MCC3 and (d) MCC4. 


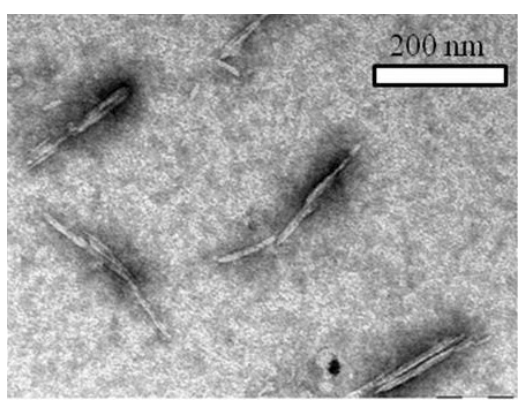

(a)

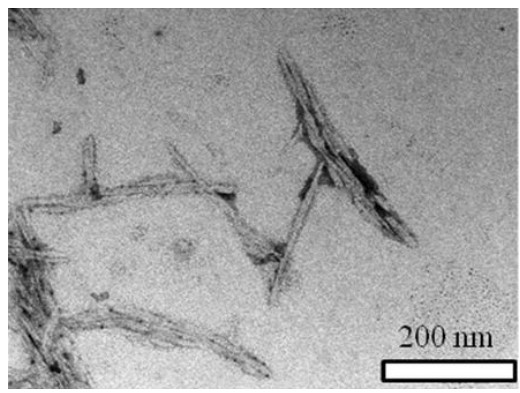

(c)

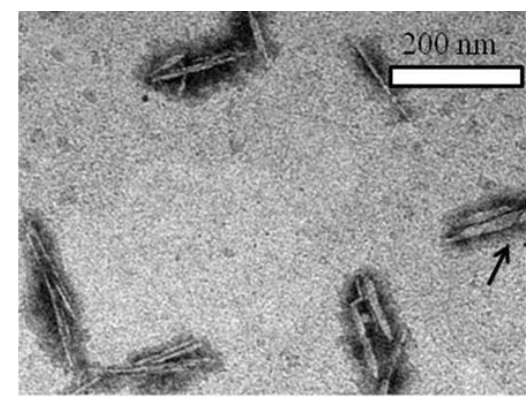

(b)

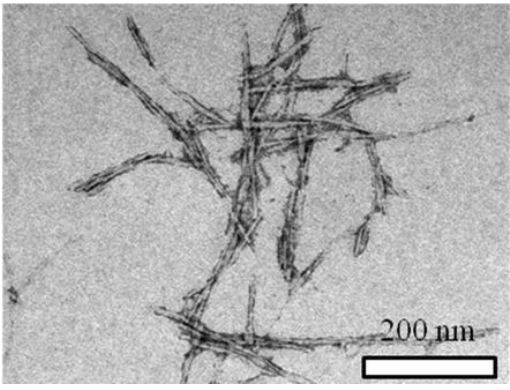

(d)

Fig. 3. TEM micrographs of the NCCs separated from the MCCs: (a) NCC1, (b) NCC2, (c) NCC3 and (d) NCC4.

electrostatic repulsion because of the negative charge of sulfate groups present on the nanocrystal surface after sulfuric acid hydrolysis, ${ }^{18}$ this association was attributed to an artificial aggregation during staining and/or drying of the specimens. Among the four micrographs of the NCCs, NCC4 agglomerated to form larger particles in the micron range compared with NCC1, NCC2 and NCC3. This result presents evidence that thinner particles are easier to assemble together.

\subsection{2. $A F M$}

Several recent reports have discussed the morphology and crystal structure of NCCs using AFM. Elazzouzi-Hafraoui et al. ${ }^{18}$ used AFM to study the cellulose surface and determine the nanoparticle thickness by topography measurements of cellulose particles isolated from cotton and tunicin. Images generated in this study using AFM illustrated the morphology and height distribution of NCCs isolated from the four MCCs. The four left-hand images are height images that represent surface topography, and the right-hand images show the particle height from the section analysis in the height image. The height images of Fig. 4 show
AFM images from NCC1 to NCC4 particles deposited onto freshly cleaved mica surfaces. Despite sonication of the suspensions performed before spreading on mica, some aggregation was observed on certain parts of the surface. Statistical analysis of the section in terms of height distribution could lead to inconclusive results, which are most probably due to particle superimposition during drying. ${ }^{18}$ The height images showed that the particle height distribution from the section analysis was calculated with a number of better-isolated particles. The height peaks of NCC1 to NCC4 were about $25.8 \mathrm{~nm}$, $19.6 \mathrm{~nm}, 23.7 \mathrm{~nm}$ and $11.5 \mathrm{~nm}$, respectively. NCC3 showed the highest peak among the four images, indicating that this sample had the most uniform particle size distribution. The height images of NCCs showed that height could typically range from $10 \mathrm{~nm}$ to $30 \mathrm{~nm}$, which is consistent with the results shown in TEM images. The order of particle height of NCCs resulted in the following trend: NCC1 > NCC3 > NCC2 > NCC4, which is consistent with the order of aggregation degree of MCCs instead of the original particle size. This result indicates that MCCs with lower aggregation degrees tend to produce narrower NCC particles during acid hydrolysis. 


\subsection{FTIR spectra}

The structure change of the polymer materials are generally determined by FTIR technology. ${ }^{25,26}$ The four MCCs and the corresponding NCCs were analyzed using FTIR to determine the various chemical changes present during hydrolysis. The FTIR spectrum of each sample is shown in Fig. 5. The FTIR spectra of the four MCCs and the corresponding NCCs were highly similar to each other. The band at $1640 \mathrm{~cm}^{-1}$ was assigned to the absorbed water. The

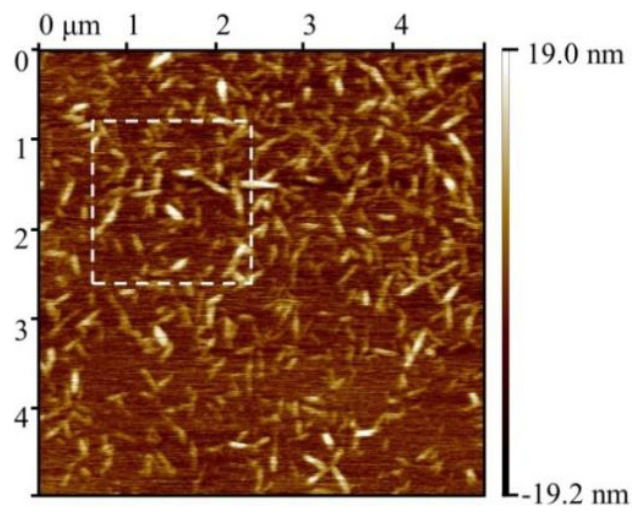

(a)

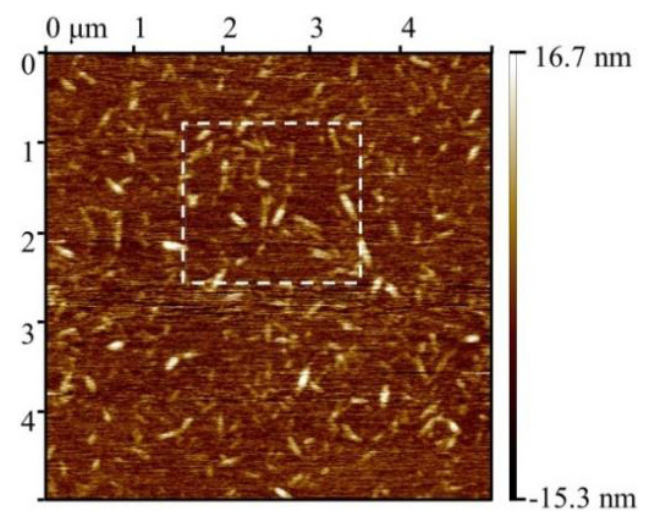

(b)

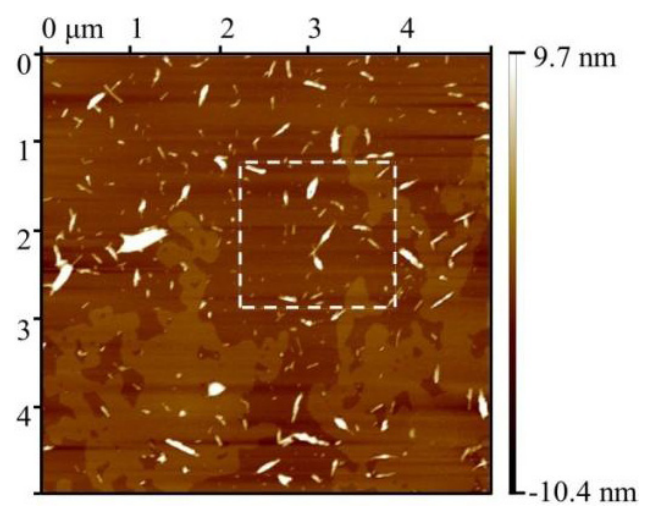

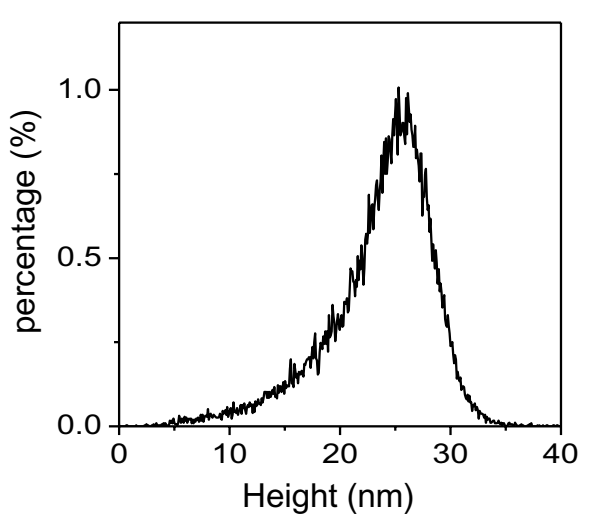
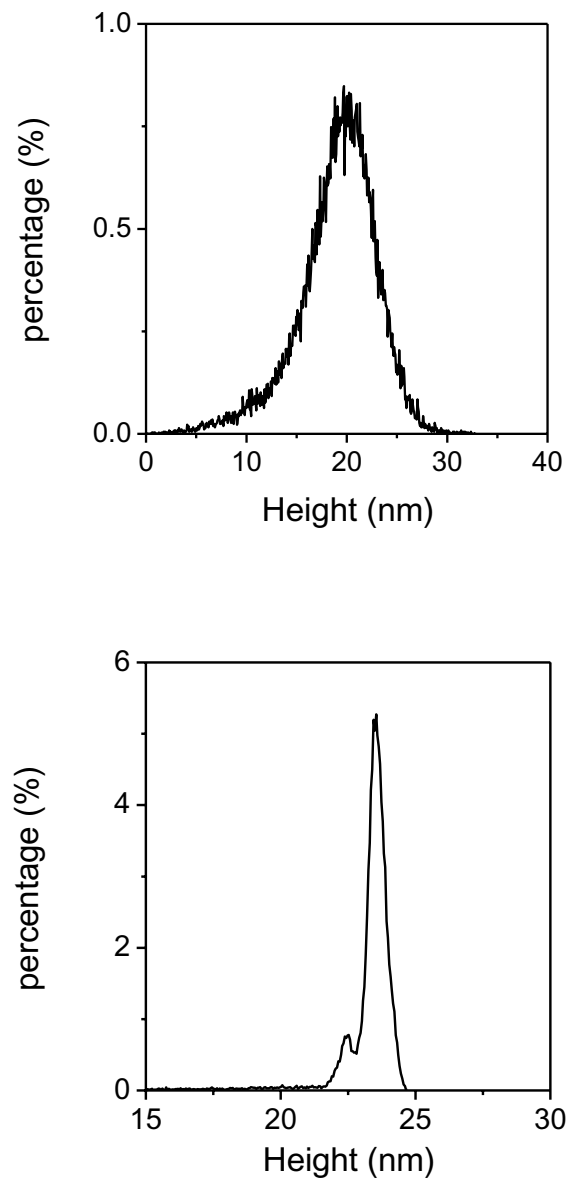

(c)

Fig. 4. AFM images and particle height distribution of NCCs: (a) NCC1, (b) NCC2, (c) NCC3 and (d) NCC4. 

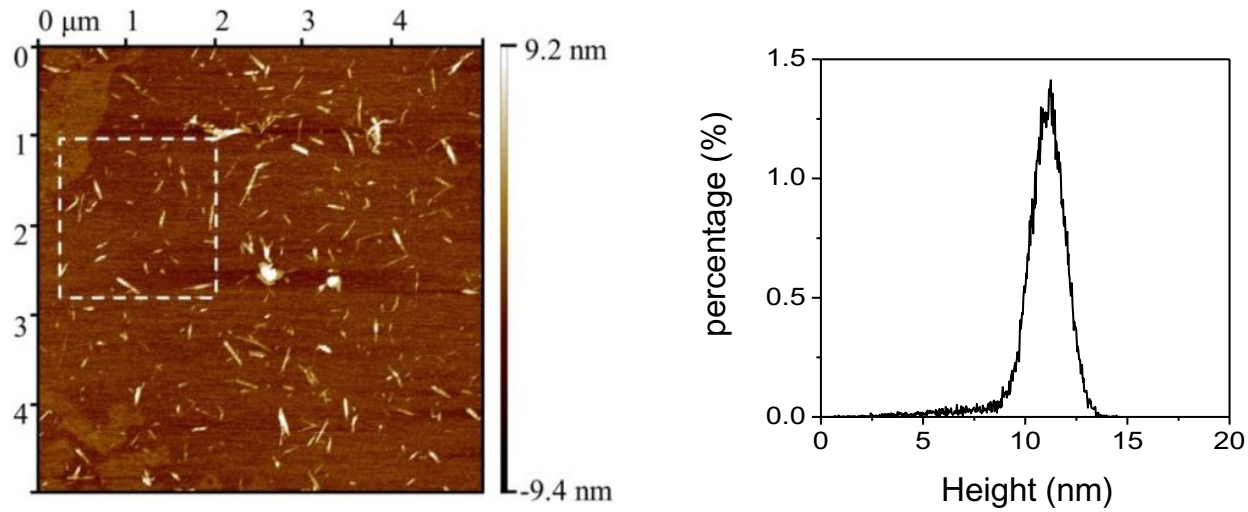

(d)

Fig. 4. (Continued)

spectra of the samples were dominated by the peaks at $3430 \mathrm{~cm}^{-1}$ to $3478 \mathrm{~cm}^{-1}$ and $1059 \mathrm{~cm}^{-1}$, reflecting the stretching vibrations of $\mathrm{O}-\mathrm{H}$ and $\mathrm{C}-\mathrm{O}$, respectively. The bands at $1061 \mathrm{~cm}^{-1}$ and $897 \mathrm{~cm}^{-1}$ are associated with $\mathrm{C}-\mathrm{O}$ stretching and $\mathrm{C}-\mathrm{H}$ deformation of glucose rings. ${ }^{8}$ The absorption peaks at 1642 ,
$1429,1376,1163$ and $1038 \mathrm{~cm}^{-1}$ are normalized with regard to the cellulose peak at $897 \mathrm{~cm}^{-1} \cdot{ }^{27}$ The peaks at around $3400 \mathrm{~cm}^{-1}$ in the spectra represent hydroxy stretching. The similar intensity of the aforementioned cellulose peaks in both of MCCs and NCCs indicated that the basic structure did not

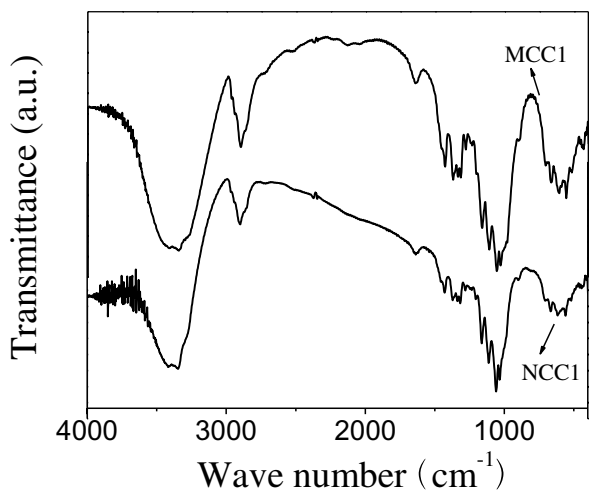

(a)

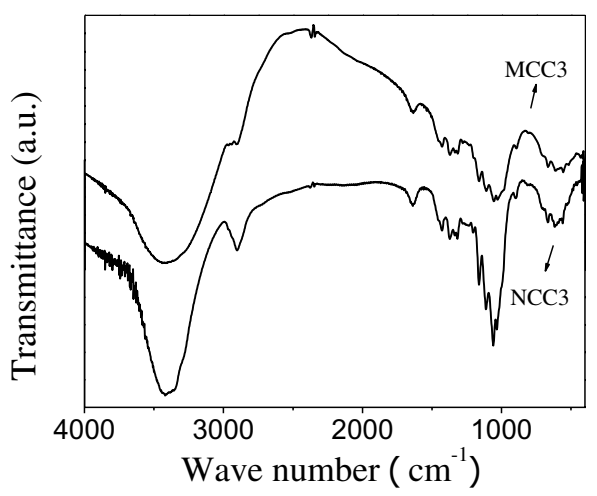

(c)

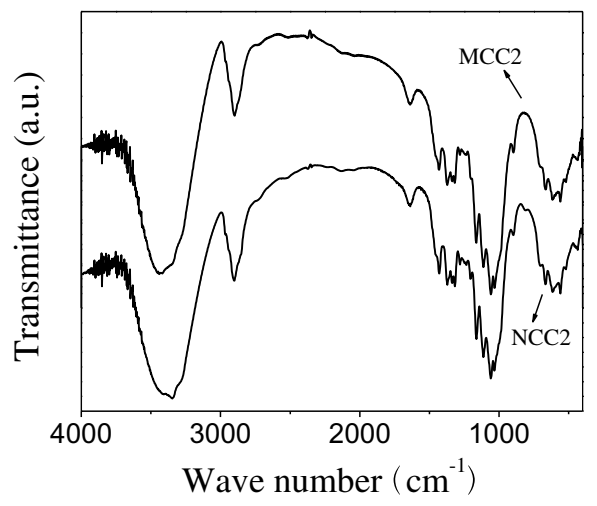

(b)

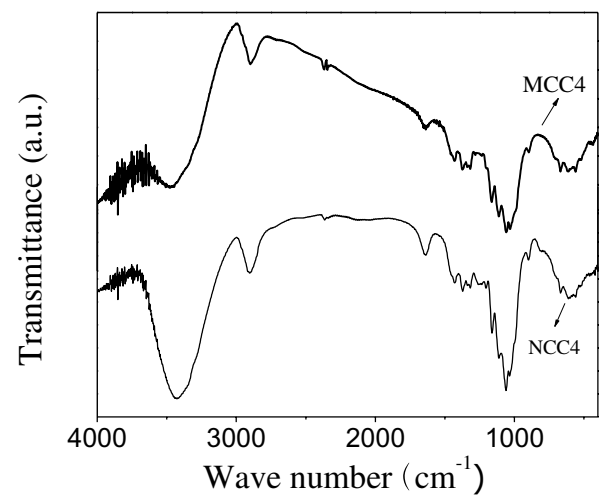

(d)

Fig. 5. FTIR spectra of MCCs and NCCs: (a) MCC1 and NCC1, (b) MCC2 and NCC2, (c) MCC3 and NCC3 and (d) MCC4 and NCC4. 


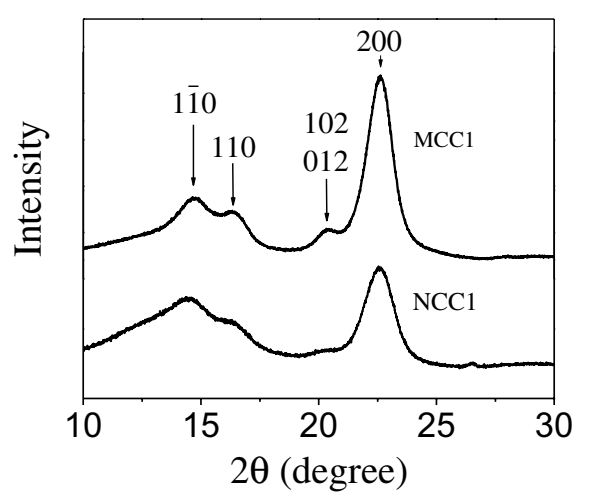

(a)

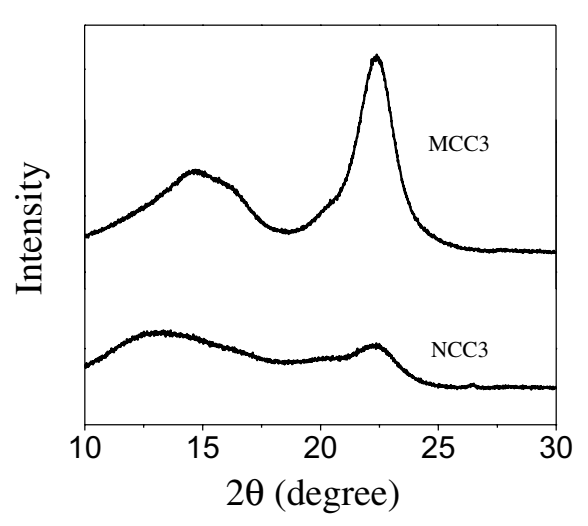

(c)

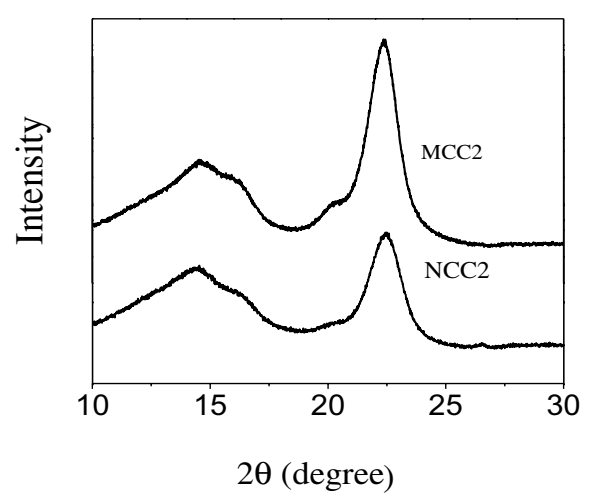

(b)

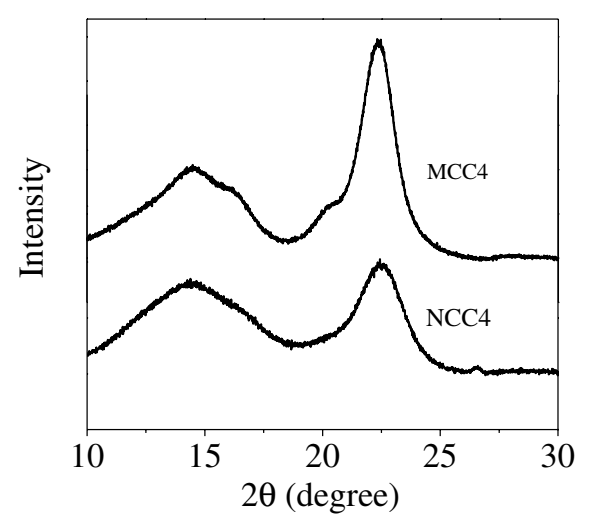

(d)

Fig. 6. XRD pattern of MCCs and NCCs: (a) MCC1 and NCC1, (b) MCC2 and NCC2, (c) MCC3 and NCC3 and (d) MCC4 and NCC4.

change during nanocrystallization. However, certain imperceptible changes could be observed by comparing the spectra of each MCC and NCC. Amorphous regions are "dissolved" by acid attack, and the absorption peak at $897 \mathrm{~cm}^{-1}$ of glucose rings decreased slightly in the NCC spectra. The shape change of the stretching peak that represents the hydroxy group was also found in the FTIR spectra. The peaks of $\mathrm{O}-\mathrm{H}$ at $3430 \mathrm{~cm}^{-1}$ to $3478 \mathrm{~cm}^{-1}$ in the spectra of four MCCs were wider and bigger than those of NCCs. After acid hydrolysis, the bands shifted to a higher frequency area, indicating the decrease of hydrogen bond during this treatment process.

\subsection{X-ray analysis}

XRD studies of the MCC and NCC fibers were performed to investigate the crystalline feature of each cellulose sample. Figure 6 and Table 2 show the XRD patterns and the crystallinities of each MCC and NCC sample. Five diffraction rings or arcs, namely
(110), (110), (102/012), (200) and (040), were recognized in all samples. These rings or arcs are characteristics of cellulose I that are based on the monoclinic indexation by Sugiyama et al. ${ }^{28}$ All patterns of the NCC samples showed peaks around $2 \theta=$ $16.5^{\circ}$ and $22.5^{\circ}$, which were speculated to represent the typical cellulose I structure, ${ }^{29}$ thus indicating that the crystal properties were not altered during hydrolysis. The crystallinities in the original four MCC fibers were $85.3 \%, 78.4 \%, 75.8 \%$ and $76.9 \%$. However, an evident decrease in crystallinity was observed in the NCC patterns. This reduction of

Table 2. Crystallinities of the MCCs and NCCs.

\begin{tabular}{lcccc}
\hline & \multicolumn{4}{c}{ Crystallinities index (\%) } \\
\cline { 2 - 5 } & 1 & 2 & 3 & 4 \\
\hline MCCs & 85.3 & 78.4 & 75.8 & 76.9 \\
NCCs & 69.5 & 64.9 & 23.7 & 48.0 \\
Reduced percentage (\%) & 18.5 & 17.2 & 68.7 & 37.6 \\
\hline
\end{tabular}


crystallinity was caused by the high concentration of sulfuric acid during nanocrystallization. The breakdown of the cellulose chains, as well as their entanglement and agglomeration, has been proven to be significantly dependent on the acid concentration used. ${ }^{3}$ The crystallinities of NCCs are reduced by $18.5 \%, 17.2 \%, 68.7 \%$ and $37.6 \%$, respectively, compared with those of relevant MCCs. The MCC3 sample was destroyed most seriously, which may be because the smallest original particles of the sample provide the highest superficial area for sulfuric attack.

\subsection{TGA}

Although the thermal properties could be studied through computational and theoretical chemistry ${ }^{30,31}$ TGA is widely used to test the thermal stability. Investigation on the thermal properties of the fibers is important to estimate their applicability for biocomposite processing, in which the processing temperature for thermoplastic polymers rises above

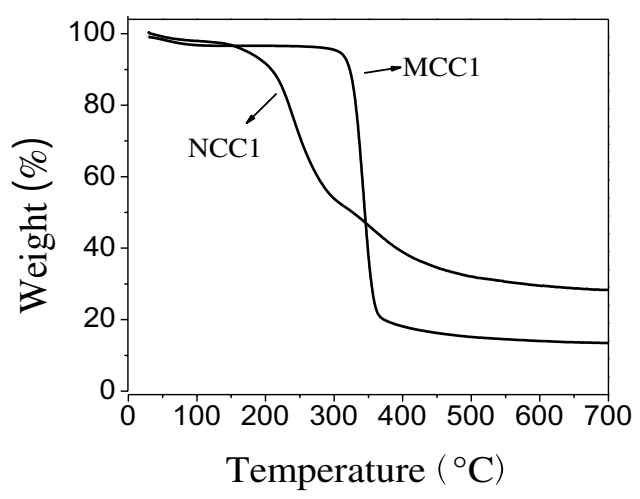

(a)

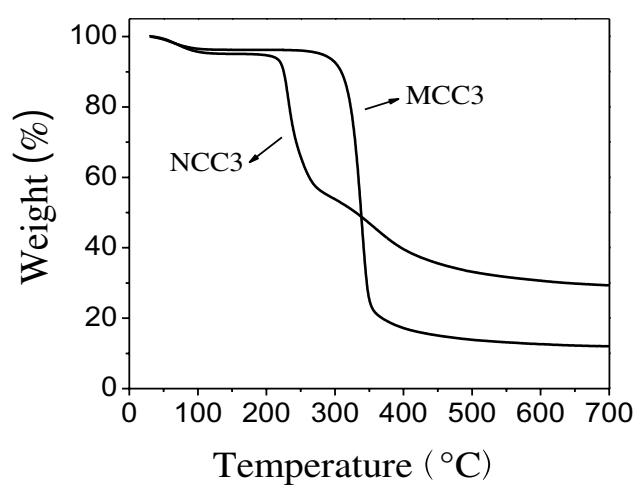

(c) $200^{\circ} \mathrm{C} .{ }^{8}$ Figure 7 shows the TGA curves of all MCCs and NCCs. All TGA curves showed an initial small drop between $50^{\circ} \mathrm{C}$ and $150^{\circ} \mathrm{C}$, which corresponded to a mass loss of approximately $5 \%$ absorbed moisture. The dominant decomposition peaks of MCCs, which were observed at around $330^{\circ} \mathrm{C}$, accounted for the pyrolysis of cellulose. In contrast to the MCC samples, we observed that the four NCCs all exhibited two stages of degradation. These results clearly illustrated that the thermal stability of the fibers decreased after hydrolysis and sonication treatments. Sonication treatment had slight effect on the thermal decomposition of nanofibers ${ }^{32}$; thus, the decrease in thermal stability was caused by the severe condition of sulfuric acid hydrolysis. Hydrolysis of fibers by $\mathrm{H}_{2} \mathrm{SO}_{4}$ is a heterogeneous reaction. ${ }^{33}$ This reaction is influenced by reaction conditions, such as acid concentration, temperature, time and mechanical agitation, as well as the physical state of the cellulose. Among all these factors, acid concentration is one of the key parameters that determine the resulting

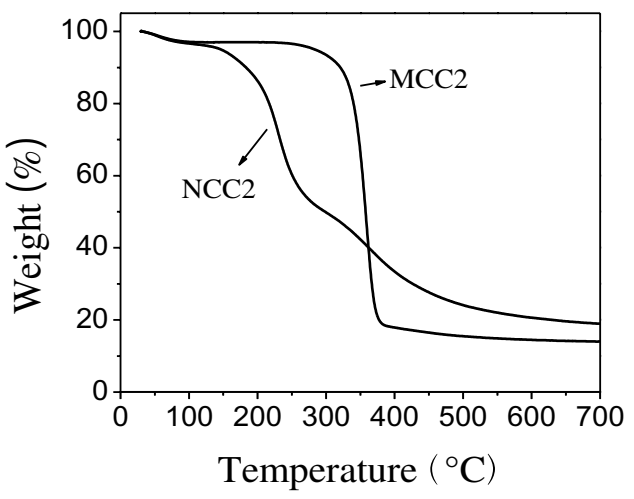

(b)

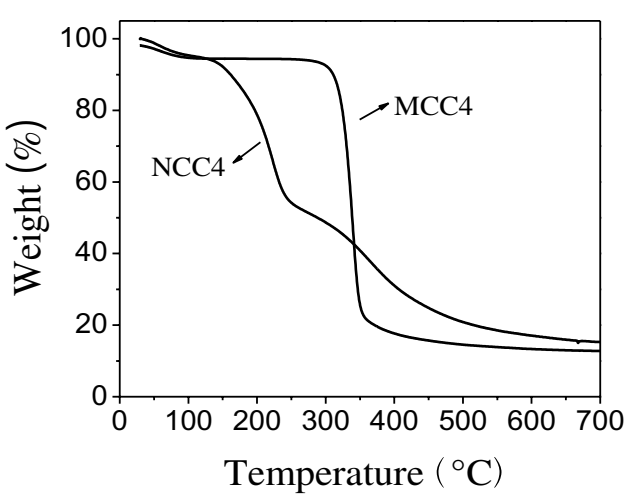

(d)

Fig. 7. TGA curves of MCCs and NCCs: (a) MCC1 and NCC1, (b) MCC2 and NCC2, (c) MCC3 and NCC3 and (d) MCC4 and NCC4. 
nature of the fibers. ${ }^{34}$ The results are highly consistent with those obtained from FTIR and XRD measurements. Compared with the other three NCCs, NCC3 showed highest degradation temperature, which was approximately $230^{\circ} \mathrm{C}$. The high thermal properties of NCC3 may broaden the fields of application of cellulose fibers, especially at temperatures higher than $200^{\circ} \mathrm{C}$ for biocomposite processing.

\section{Conclusion}

NCCs were separated from four commercial MCCs by sulfuric acid hydrolysis and then sonicated. The morphology and particle size of MCCs were characterized through SEM and laser particle size analyzer. The results showed different shapes and sizes, and the aggregation degree followed the order MCC1 > MCC3 > MCC2 > MCC4. The resulting NCCs were composed of elongated rods with $100 \mathrm{~nm}$ to $250 \mathrm{~nm}$ in length and $10 \mathrm{~nm}$ to $30 \mathrm{~nm}$ in width. The same order of NCC height as the aggregation degree of MCCs indicates that MCCs with lower aggregation degree tend to produce narrower NCC particles during acid hydrolysis process, which were easier to assemble together. Besides the morphology and particle size of NCCs characterized by TEM and AFM, the molecular structure, crystallinity and thermal properties of the nanosized particles were also investigated. The FTIR and XRD results showed that the basic structure and crystal properties of MCCs did not change during the preparation process. However, the harsh hydrolysis condition reduced their crystallinity and decreased the thermal stability to varying degrees. MCC3, which presented the lowest original particle size and good dispersity, produced the most uniform and thermally stable NCC. This result suggests that both particle size and dispersity are key parameters that influence the separation of NCCs.

\section{Acknowledgments}

We gratefully acknowledge funding support for this work provided by The National High-Tech R\&D Program (863 Program) for the 12th Five-Year Plan (2011AA100503) and The Agricultural Science and Technology Innovation Program (ASTIP) of Chinese Academy of Agricultural Sciences. This work was also supported by Basic Scientific
Research Foundation of National non-Profit Scientific Institute of China (BSRF201404).

\section{References}

1. M. Deng et al., Mater. Lett. 63, 1851 (2009).

2. E. M. Teixeira et al., Carbohydr. Polym. 78, 422 (2009).

3. K. Das et al., Cellulose 16, 783 (2009).

4. T. Virtanen et al., Cellulose 19, 219 (2012).

5. M. S. Jahan et al., Cellulose 18, 451 (2011).

6. R. L. de Oliveira et al., J. Therm. Anal. Calorim. 106, 703 (2011).

7. F. F. Morehead, Text. Res. J. 20, 549 (1950).

8. A. Alemdar and M. Sain, Bioresour. Technol. 99, 1664 (2008).

9. W. Chen et al., Cellulose 18, 433 (2011).

10. R. H. Marchessault, F. F. M. Morehead and M. J. Koch, J. Colloid. Sci. 16, 327 (1961).

11. S. M. Mukherjee and H. J. Woods, Biochim. Biophys. Acta 10, 499 (1953).

12. M. A. Herrera, A. P. Mathew and K. Oksman, Mater. Lett. 71, 28 (2012).

13. D. Bondeson, A. Mathew and K. Oksman, Cellulose 13, 171 (2006).

14. X. M. Dong, J. F. Revol and G. D. Gray, Cellulose 5, 19 (1998).

15. J. Araki and S. Kuga, Langmuir 17, 4493 (2001).

16. E. Belamie, P. Davidson and M. M. Giraud-Guille, J. Phys. Chem. B 108, 14991 (2004).

17. J. F. Revol et al., Int. J. Biol. Macromol. 14, 170 (1992).

18. S. Elazzouzi-Hafraoui et al., Biomacromolecules 9, 57 (2007).

19. D. Bhattacharya, L. T. Germinario and W. T. Winter, Carbohydr. Polym. 73, 371 (2008).

20. L. G. J. M. A. Segal et al., Text. Res. J. 29, 786 (1959).

21. X. Chen, K. Ding and N. Ayres, Polym. Chem. 2, 2635 (2011).

22. X. Chen and N. Ayres, Macromolecules 43, 1341 (2010).

23. X. Chen and N. Ayres, J. Polym. Sci., A Polym. Chem. 49, 3030 (2011).

24. K. Abe and H. Yano, Cellulose 17, 271 (2010).

25. Y. Huang et al., J. Polym. Sci., A Polym. Chem. 51, 5230 (2013).

26. L. Taylor, X. Chen and N. Ayres, Polym. Int. 63, 127 (2014).

27. M. F. Rosa et al., Carbohydr. Polym. 81, 83 (2010).

28. J. Sugiyama, R. Vuong and H. Chanzy, Macromolecules 24, 4168 (1991).

29. Y. Nishiyama, P. Langan and H. Chanzy, J. Am. Chem. Soc. 124, 9074 (2002). 
30. G. W. Wang, X. P. Chen and X. Cheng, Chem.-Eur. J. 12, 7246 (2006).

31. P. $\mathrm{Wu}$ et al., Comput. Theor. Chem. 1030, 67 (2014).

32. D. Klemm et al., Comprehensive Cellulose Chemistry, Vol. 1: Fundamentals and Analytical Methods (Wiley-VCH publication, Weinheim), 1998).
33. Q. Xiang et al., Appl. Biochem. Biotechnol. 105, 505 (2003).

34. M. A. Saïd Azizi Samir et al., Macromolecules 37, 4313 (2004). 